\title{
TANGUY RIVOAL
}

\section{Séries hypergéométriques et irrationalité des valeurs de la fonction zêta de Riemann}

\author{
Journal de Théorie des Nombres de Bordeaux, tome 15, $\mathrm{n}^{\circ} 1$ (2003), \\ p. 351-365 \\ <http://www.numdam.org/item?id=JTNB_2003_15_1_351_0>
}

(C) Université Bordeaux 1, 2003, tous droits réservés.

L'accès aux archives de la revue «Journal de Théorie des Nombres de Bordeaux » (http://jtnb.cedram.org/) implique l'accord avec les conditions générales d'utilisation (http://www.numdam.org/conditions). Toute utilisation commerciale ou impression systématique est constitutive d'une infraction pénale. Toute copie ou impression de ce fichier doit contenir la présente mention de copyright.

\section{Numdam}

Article numérisé dans le cadre du programme

Numérisation de documents anciens mathématiques

http://www.numdam.org/ 


\title{
Séries hypergéométriques et irrationalité des valeurs de la fonction zêta de Riemann
}

\author{
par TANGUY RIVOAL
}

\begin{abstract}
RÉSUMÉ. Nous effectuons un survol des résultats connus sur la nature diophantienne des valeurs de la fonction zêta de Riemann aux entiers. Nous mettons en particulier l'accent sur le rôle important des séries hypergéométriques dans les démonstrations de l'irrationalité de $\zeta(2), \zeta(3)$ et d'une infinité des nombres $\zeta(2 n+1)$.

ABSTRACT. In this survey, we present some known results in diophantine theory of values of the Riemann zeta function at integers. In particular, we will emphasise the importance of hypergeometric series in the proofs of the irrationality of $\zeta(2), \zeta(3)$ and of infinitely many $\zeta(2 n+1) s$.
\end{abstract}

\section{Introduction}

Nous effectuons un tour d'horizon de résultats anciens et récents concernant la nature arithmétique des valeurs pour $s$ entier $\geq 2$ de la fonction $\zeta(s)=\sum_{n>1} 1 / n^{s}$. En notant $B_{2 n}$ les nombres (rationnels) de Bernoulli, la formule d'Euler

$$
\zeta(2 n)=(-1)^{n-1} \frac{2^{2 n-1} B_{2 n}}{(2 n) !} \pi^{2 n}
$$

et la transcendance de $\pi$ par Lindemann [Li] prouvent la transcendance de tous les $\zeta$ pairs. En revanche, une telle formule d'Euler fait défaut pour les $\zeta$ impairs et il a fallu attendre 1978 pour voir la démonstration de l'irrationalité de $\zeta(3)$ (et aussi de $\zeta(2)$ ) par Apéry [Ap1,2]. Sa méthode fit plus d'un sceptique mais fut finalement acceptée après les travaux de Beukers [Be1], Cohen [Coh], Van der Poorten [VdP]. Pourtant aucune des nombreuses approches proposées depuis n'a permis de prouver l'irrationalité d'un autre $\zeta$ impair.

En 2000, l'auteur du présent survol, en généralisant une construction de $\mathrm{K}$. Ball $[\mathrm{Ba}]$, est parvenu à démontrer l'irrationalité d'une infinité de $\zeta$ impairs (cf. [Ril] ou aussi $[\mathrm{BR}]$ ), mais sans pouvoir en citer un autre

Manuscrit reçu le 19 novembre 2001. 
que $\zeta(3)$. Néanmoins, en 2001, il a pu montrer que l'une au moins des neuf valeurs $\zeta(5), \zeta(7), \ldots, \zeta(21)$ est irrationnelle [Ri2]. Ce résultat a été rapidement amélioré par Zudilin [Zu2] qui a montré l'irrationalité d'au moins un des quatre nombres $\zeta(5), \zeta(7), \zeta(9), \zeta(11)$. L'irrationalité de $\zeta(5)$ est donc encore incertaine.

Tous ces résultats peuvent être placés dans le contexte récent et plus général des séries mutizêtas (cf. [Wa] pour une introduction)

$$
\zeta\left(s_{1}, \ldots, s_{k}\right)=\sum_{1 \leq n_{k}<\cdots<n_{1}} \frac{1}{n_{1}^{s_{1}} \cdots n_{k}^{s_{k}}}
$$

où $s_{1} \geq 2$ et $s_{2}, \ldots, s_{k} \geq 1$ sont entiers. La détermination des relations algébriques possibles entre des analogues symboliques de ces nombres laisse espérer l'indépendance algébrique sur $\mathbb{Q}$ de $\pi, \zeta(3), \zeta(5), \zeta(7)$, etc. On mesure le chemin à parcourir.

\section{Notations}

Toutes les démonstrations proposées dans la suite peuvent être placées dans le cadre de la théorie des fonctions hypergéométriques

$$
{ }_{p+1} F_{p}\left(\begin{array}{c}
a_{1}, a_{2}, \ldots, a_{p+1} \\
b_{1}, b_{2}, \ldots, b_{p}
\end{array} \mid z\right)=\sum_{k=0}^{+\infty} \frac{\left(a_{1}\right)_{k}\left(a_{2}\right)_{k} \ldots\left(a_{p+1}\right)_{k}}{(1)_{k}\left(b_{1}\right)_{k}\left(b_{2}\right)_{k} \ldots\left(b_{p}\right)_{k}} z^{k}
$$

où $p$ est un entier $\geq 1,(\alpha)_{0}=1$ et $(\alpha)_{k}=\alpha(\alpha+1) \cdots(\alpha+k-1)$ définissent le symbole de Pochhammer, $a_{i} \in \mathbb{C}, b_{j} \in \mathbb{C} \backslash\{0,-1,-2, \ldots\}$ et $z \in \mathbb{C},|z|<1$, avec parfois convergence sur le cercle $|z|=1$.

Les séries introduites dans la suite sont construites pour donner des combinaisons linéaires à coefficients polynomiaux des fonctions polylogarithmes

$$
\operatorname{Li}_{s}(z)=\sum_{n=1}^{+\infty} \frac{z^{k}}{n^{s}} \quad(s \geq 1,|z| \geq 1)
$$

et donc des valeurs $\zeta(s)$ par spécialisation en $z= \pm 1$. Ces approximations proviennent de la théorie des approximants de Padé. Comme on le verra, ce lien est bien connu dans le cas de $\zeta(2)$ et de $\zeta(3)$ (cf. [Be2,3], [Hu], [Ni], [Pr] et [So] par exemple).

Les démonstrations sont plus particulièrement centrées sur le comportement asymptotique des séries. Il sera fait usage de la méthode du col dont voici un énoncé possible (cf. [Cop, pp 91-94] ou [Di, pp279-285]).

Méthode du col Soit $g$ et $w$ deux fonctions analytiques dans un ouvert simplement connexe $\mathcal{D} d u$ plan. Supposons qu'il existe $z_{0} \in \mathcal{D}$ tel que $w^{\prime}\left(z_{0}\right)=0$ et $w^{\prime \prime}\left(z_{0}\right)=\left|w^{\prime \prime}\left(z_{0}\right)\right| e^{i \alpha_{0}} \neq 0$. Si $L$ est un chemin inclus dans $\mathcal{D}$ 
le long duquel $w(z)$ admet un maximum global en $z_{0}$, alors

$$
\int_{L} g(z) e^{n w(z)} \mathrm{d} z \sim g\left(z_{0}\right) \sqrt{\frac{2 \pi}{n\left|w^{\prime \prime}\left(z_{0}\right)\right|}} e^{i\left( \pm \frac{\pi}{2}-\frac{\alpha_{0}}{2}\right)} e^{n w\left(z_{0}\right)} \quad(n \rightarrow+\infty)
$$

où le choix de \pm dépend de l'orientation de $L$.

Les estimations arithmétiques des dénominateurs des combinaisons linéaires sont données sans plus de formalité : de très importantes et difficiles questions sont cependant soulevées par ces dénominateurs.

Enfin, fixons quelques points de notation : $\log (z)$ sera considéré avec sa détermination principale, $n$ désignera toujours un entier $\geq 0$ et $d_{n}$ le plus petit commun multiple des nombres $1,2, \ldots, n$. La formule $d_{n}=$ $\prod_{p \text { ler }} p^{[\log (n) / \log (p)]}$ et le théorème des nombres premiers justifient que

$$
\lim _{n \rightarrow+\infty} d_{n}^{1 / n}=e .
$$

\section{Une démonstration exemplaire}

Beukers [Be2] (cf. également $[\mathrm{Hu}]$ ) a montré que la démonstration d'Apéry de l'irrationalité de $\zeta(2)$ revenait à résoudre le problème suivant : déterminer des polynômes $A_{n}(z), B_{n}(z)$ et $C_{n}(z)$ de degré au plus $n$ et tels que l'on ait les approximations au voisinage de $\infty$ et de 1

$$
S_{n}(z):=A_{n}(z) \mathrm{Li}_{2}(1 / z)+B_{n}(z) \operatorname{Li}_{1}(1 / z)+C_{n}(z)=\mathrm{O}\left(1 / z^{n+1}\right)
$$

et

$$
A_{n}(z) \log (z)+B_{n}(z)=\mathrm{O}\left((1-z)^{n+1}\right) .
$$

Ce problème admet une solution unique à une constante multiplicative près et on a

$$
S_{n}(z)=n ! \sum_{k=1}^{+\infty} \frac{(k-1) \cdots(k-n)}{k^{2}(k+1)^{2} \cdots(k+n)^{2}} z^{-k},
$$

série qui converge pour $z$ complexe, $|z| \geq 1$. En décomposant en éléments simples le terme rationnel de $S_{n}(z)$, on montre que

$$
A_{n}(z)=\sum_{k=0}^{n}(-1)^{n-k}\left(\begin{array}{l}
n \\
k
\end{array}\right)^{2}\left(\begin{array}{c}
n+k \\
n
\end{array}\right) z^{k} \in \mathbb{Z}[z]
$$

et $d_{n} B_{n}(z) \in \mathbb{Z}[z], d_{n}^{2} C_{n}(z) \in \mathbb{Z}[z]$. De plus la convergence de $S_{n}(1)$ et la divergence de $\mathrm{Li}_{1}(1)$ impliquent que $\left.B_{n}(1)=0{ }^{1}\right)$. Donc $d_{n}^{2} S_{n}(1)=$ $d_{n}^{2} A_{n}(1) \zeta(2)+d_{n}^{2} C_{n}(1) \in \mathbb{Z} \zeta(2)+\mathbb{Z}$.

Pour prouver l'irrationalité de $\zeta(2)$, il suffit de montrer que $S_{n}(1) \neq 0$ $\left({ }^{2}\right)$ et que $d_{n}^{2} S_{n}(1)$ tend vers 0 , en vertu du lemme élémentaire suivant :

\footnotetext{
${ }^{1}$ Cela se voit aussi sur la condition $A_{n}(z) \log (z)+B_{n}(z)=\mathrm{O}\left((1-z)^{n+1}\right)$ en $z=1$.

${ }^{2}$ au moins pour une infinité d'entiers $n$.
} 
Lemme 1. Soit $\alpha$ un nombre réel. Supposons qu'il existe deux suites d'entiers $\left(p_{n}\right)_{n \geq 0}$ et $\left(q_{n}\right)_{n \geq 0}$ telles que pour une infinité d'entiers $n$, on ait $q_{n} \alpha-p_{n} \neq \overline{0}$ et $\lim _{n \rightarrow+\infty}\left(q_{n} \alpha-p_{n}\right)=0$. Alors $\alpha$ est irrationnel.

La non-nullité de $S_{n}(1)$ est ici claire puisque les $n$ premiers termes de $S_{n}(1)$ sont nuls et tous les suivants strictement positifs. L'estimation de la décroissance peut être faite de plusieurs façons : nous utilisons ici des intégrales multiples et indiquerons d'autres méthodes dans les paragraphes suivants. Remarquons que la série $S_{n}(z)$ est une fonction hypergéométrique

$$
S_{n}(z)=z^{-n-1} \frac{\Gamma(n+1)^{4}}{\Gamma(2 n+2)^{2}}{ }_{3} F_{2}\left(\begin{array}{c}
n+1, n+1, n+1 \\
2 n+2,2 n+2
\end{array} \mid z^{-1}\right)
$$

dont les paramètres sont tels que l'on peut lui appliquer l'identité intégrale d'Euler convenablement itérée [Sl, p. 108, 4.1.2] :

$$
S_{n}(z)=\int_{0}^{1} \int_{0}^{1}\left(\frac{x(1-x) y(1-y)}{z-x y}\right)^{n} \cdot \frac{\mathrm{d} x \mathrm{~d} y}{z-x y}
$$

ce qui, en $z=1$, n'est rien d'autre que l'intégrale de Beukers pour $\zeta(2)$. On en déduit alors que

$\lim _{n \rightarrow+\infty}\left(d_{n}^{2} S_{n}(1)\right)^{1 / n}=e^{2} \max _{(x, y) \in[0,1]^{2}}\left(\frac{x(1-x) y(1-y)}{1-x y}\right)=e^{2}\left(\frac{\sqrt{5}-1}{2}\right)^{5}<1$.

D'où le :

Théorème 1. $\zeta(2)$ est irrationnel.

\section{Le théorème d'Apéry}

Nous esquissons une démonstration de l'irrationalité de $\zeta(3)$ due à Nesterenko [Ne2], poursuivant une approche de Gutnik [Gu] et Beukers [Be3]. Ce dernier résout le problème de Padé suivant : déterminer des polynômes $A_{n}(z), B_{n}(z), C_{n}(z), D_{n}(z)$ de degré au plus $n$ tels que $B_{n}(1)=0$,

$$
U_{n}(z):=A_{n}(z) \operatorname{Li}_{2}(1 / z)+B_{n}(z) \operatorname{Li}_{1}(1 / z)+C_{n}(z)=\mathrm{O}\left(1 / z^{n+1}\right)
$$

et

$$
V_{n}(z):=2 A_{n}(z) \operatorname{Li}_{3}(1 / z)+B_{n}(z) \mathrm{Li}_{2}(1 / z)+D_{n}(z)=\mathrm{O}\left(1 / z^{n+1}\right) .
$$

Il montre que ce problème a une solution unique, à une constante multiplicative près, donnée par les deux séries convergentes pour $z$ complexe, $|z| \geq 1$ :

$$
U_{n}(z)=\sum_{k=1}^{+\infty}\left(\frac{(k-1) \cdots(k-n)}{k(k+1) \cdots(k+n)}\right)^{2} z^{-k}
$$

et

$$
V_{n}(z)=-\sum_{k=1}^{+\infty} \frac{\mathrm{d}}{\mathrm{d} k}\left(\frac{(k-1) \cdots(k-n)}{k(k+1) \cdots(k+n)}\right)^{2} z^{-k}
$$


On a également

$$
A_{n}(z)=\sum_{k=0}^{n}\left(\begin{array}{l}
n \\
k
\end{array}\right)^{2}\left(\begin{array}{c}
n+k \\
n
\end{array}\right)^{2} z^{k} \in \mathbb{Z}[z]
$$

et $d_{n} B_{n}(z) \in \mathbb{Z}[z], d_{n}^{2} C_{n}(z) \in \mathbb{Z}[z], d_{n}^{3} D_{n}(z) \in \mathbb{Z}[z]$.

On a donc $d_{n}^{3} V_{n}(1)=2 d_{n}^{3} A_{n}(1) \zeta(3)+d_{n}^{3} D_{n}(1) \in \mathbb{Z} \zeta(3)+\mathbb{Z}$. Pour conclure, il suffit maintenant de montrer que $V_{n}(1) \neq 0\left({ }^{3}\right)$ et que $d_{n}^{3} V_{n}(1)$ tend vers 0 . Or ces deux points ne sont absolument pas évidents ! S'il existe bien une intégrale de Beukers

$$
V_{n}(1)=\int_{0}^{1} \int_{0}^{1} \int_{0}^{1}\left(\frac{x(1-x) y(1-y) z(1-z)}{1-(1-x y) z}\right)^{n} \cdot \frac{\mathrm{d} x \mathrm{~d} y \mathrm{~d} z}{1-(1-x y) z}
$$

il semble qu'il n'existe pas de démonstration directe de ce fait à partir de la série qui définit $V_{n}(1)$. On peut néanmoins faire les remarques suivantes. La série $z^{-n-1} U_{n}(1 / z)$ est une fonction hypergéométrique

$$
\frac{\Gamma(n+1)^{4}}{\Gamma(2 n+2)^{2}}{ }_{4} F_{3}\left(\begin{array}{c}
n+1, n+1, n+1, n+1 \\
1,2 n+2,2 n+2
\end{array} \mid z\right)
$$

vérifiant la même équation différentielle hypergéométrique que la fonction $z^{-n-1}\left(V_{n}(1 / z)-\log (z) U_{n}(1 / z)\right):$

$$
\vartheta^{2}(\vartheta+2 n+1)^{2} y-z(\vartheta+n+1)^{4} y=0
$$

où $\vartheta=z \mathrm{~d} / \mathrm{d} z$. Nørlund (cf. [Nø, p.316]) montre que les solutions de telles équations différentielles peuvent toujours être représentées par des intégrales complexes, dites de Barnes (cf. [Sl, chapitre 4]). Dans le cas présent, on obtient

$$
\begin{array}{r}
V_{n}(z)+\log (z) U_{n}(z)=\frac{1}{2 i \pi} \int_{L}\left(\frac{(s-1) \cdots(s-n)}{s(s+1) \cdots(s+n)}\right)^{2}\left(\frac{\pi}{\sin (\pi s)}\right)^{2} z^{s} \mathrm{~d} s \\
=\frac{1}{2 i \pi} \int_{L}\left(\frac{\Gamma(n+1-s) \Gamma(s)^{2}}{\Gamma(n+1+s)}\right)^{2} z^{s} \mathrm{~d} s
\end{array}
$$

où $z$ est un nombre complexe tel que $|\arg (z)|<2 \pi, L$ est une droite verticale orientée de $-i \infty$ à $+i \infty$ incluse dans la bande $0<\operatorname{Re}(s)<n$. Nesterenko justifie à l'aide de la formule de Stirling que

$$
V_{n}(1)=\frac{2 \pi}{n i} \int_{c-i \infty}^{c+i \infty} \frac{1+t}{(1-t) t^{2}} e^{2(n+1) f(t)} \mathrm{d} t \cdot\left(1+\mathrm{O}\left(n^{-1}\right)\right)
$$

avec $c$ réel quelconque dans $] 0,1[$ et $f(t)=(1-t) \log (1-t)+2 t \log (t)-$ $(1+t) \log (1+t)$. En appliquant la méthode du col avec le choix $c=1 / \sqrt{2}$,

\footnotetext{
${ }^{3}$ cf. note (2).
} 
il en déduit que

$$
\lim _{n \rightarrow+\infty}\left|d_{n}^{3} V_{n}(1)\right|^{1 / n}=e^{3}(\sqrt{2}-1)^{4}<1
$$

ce qui prouve le célèbre résultat d'Apéry :

Théorème 2. $\zeta(3)$ est irrationnel.

\section{Une série remarquable}

Nesterenko [ $\mathrm{Ne} 2$, p. 629] note que sa méthode ne peut pas être considérée comme élémentaire (à cause de la méthode du col) et pose le problème de trouver une telle démonstration. Dans ce but, $\mathrm{K}$. Ball [Ba] a construit une série $B_{n}(1)$ où pour tout $z$ complexe, $|z| \geq 1$,

$$
\begin{array}{r}
B_{n}(z):=\sum_{k=1}^{+\infty} n !^{2}\left(k+\frac{n}{2}\right) \frac{(k-1) \cdots(k-n)(k+n+1) \cdots(k+2 n)}{k^{4}(k+1)^{4} \cdots(k+n)^{4}} z^{-k} \\
=\beta_{n}(z) \operatorname{Li}_{4}(1 / z)+\alpha_{n}(z) \operatorname{Li}_{3}(1 / z) \\
+\gamma_{n}(z) \operatorname{Li}_{2}(1 / z)+\kappa_{n}(z) \operatorname{Li}_{1}(1 / z)+\delta_{n}(z)
\end{array}
$$

avec

$$
\beta_{n}(z)=(-1)^{n} \sum_{k=0}^{n}\left(\begin{array}{l}
n \\
k
\end{array}\right)^{4}\left(\begin{array}{c}
n+k \\
n
\end{array}\right)\left(\begin{array}{c}
2 n-k \\
n
\end{array}\right)\left(\frac{n}{2}-k\right) z^{k} \in \mathbb{Z}[z],
$$

et $d_{n} \alpha_{n}(z) \in \mathbb{Z}[z], d_{n}^{2} \gamma_{n}(z) \in \mathbb{Z}[z], d_{n}^{3} \kappa_{n}(z) \in \mathbb{Z}[z], d_{n}^{4} \delta_{n}(z) \in \mathbb{Z}[z]$. De plus, par convergence, $\kappa_{n}(1)=0$. On a donc a priori

$$
B_{n}(1)=\beta_{n}(1) \zeta(4)+\alpha_{n}(1) \zeta(3)+\gamma_{n}(1) \zeta(2)+\delta_{n}(1)
$$

ce qui n'est pas très intéressant. Or $B_{n}(1)$ possède une très remarquable propriété : $\beta_{n}(1)=\gamma_{n}(1)=0$ et donc $d_{n}^{4} B_{n}(1)=d_{n}^{4} \alpha_{n}(1) \zeta(3)+d_{n}^{4} \delta_{n}(1) \in$ $\mathbb{Z} \zeta(3)+\mathbb{Z}$.

Il est clair, comme pour la série $S_{n}(1)$, que $B_{n}(1) \neq 0\left({ }^{4}\right)$. On peut estimer $d_{n}^{4} B_{n}(1)$ directement à l'aide de la formule de Stirling (cf. [BR, seconde démonstration du lemme 3]). On peut aussi remarquer que $B_{n}(z)$ est une série hypergéométrique, dite "well-poised" dans la terminologie de $[\mathrm{Sl}]:$

$$
\begin{aligned}
& B_{n}(z)=z^{-n-1} \frac{\Gamma(n+1)^{7} \Gamma(3 n+3)}{2 \Gamma(2 n+2)^{5}} \\
& \quad \times{ }_{7} F_{6}\left(\begin{array}{r}
3 n+2,3 n / 2+2, n+1, \ldots, n+1 \\
3 n / 2+1,2 n+2, \ldots, 2 n+2
\end{array} \mid z^{-1}\right) .
\end{aligned}
$$

${ }^{4}$ Il n'y a donc pas lieu de renvoyer vers la note (2). 
Comme au paragraphe 2 , on en déduit la représentation intégrale eulérienne suivante :

$$
B_{n}(1)=\frac{(3 n+1) !}{n !^{3}} \int_{[0,1]^{5}}\left(\frac{\prod_{l=1}^{5} x_{l}\left(1-x_{l}\right)}{\left(1-x_{1} \cdots x_{5}\right)^{3}}\right)^{n} \cdot \frac{1+x_{1} \cdots x_{5}}{\left(1-x_{1} \cdots x_{5}\right)^{2}} \cdot \prod_{l=1}^{5} \mathrm{~d} x_{l}
$$

dont découle que

$$
\lim _{n \rightarrow+\infty}\left(d_{n}^{4} B_{n}(1)\right)^{1 / n}=e^{4}(\sqrt{2}-1)^{4}>1 .
$$

La suite $d_{n}^{4} B_{n}(1)$ ne tend donc malheureusement pas vers 0 et on ne redémontre pas l'irrationalité de $\zeta(3)$.

\section{Indépendance linéaire d'une infinité de zêta impairs}

La conséquence la plus intéressante de la forme particulière de $B_{n}(1)$ est la disparition de $\zeta(2)$ et $\zeta(4)$. Dans [Ri1], l'auteur a introduit la généralisation suivante de $B_{n}(z)$, convergente pour $z$ complexe, $|z| \geq 1$ :

$$
\begin{aligned}
S_{n, a, r}(z) & :=n !^{a-2 r} \sum_{k=1}^{+\infty} \frac{(k-r n)_{r n}(k+n+1)_{r n}}{(k)_{n+1}^{a}} z^{-k} \\
& =P_{0, n}(z)+\sum_{l=1}^{a} P_{l, n}(z) \operatorname{Li}_{l}(1 / z) .
\end{aligned}
$$

où les $P_{l, n}(z)$ sont des polynômes de degré au plus $n$ et les paramètres $a$ et $r$ sont des entiers tels que $1 \leq r<a / 2$. L'introduction du paramètre $r$ permet d'avoir un contrôle plus souple de la "taille" de $S_{n, a, r}(z)$ et des $P_{l, n}(z)$. Comme espéré, si $n$ est pair et $a$ impair $\geq 3$, alors $P_{2 l, n}(1)=0$ : cela résulte de la formule de \pm -réciprocité

$$
z^{n} P_{l, n}(1 / z)=(-1)^{(n+1) a+l} P_{l, n}(z) .
$$

Donc

$$
S_{n, a, r}(1)=P_{0, n}(1)+\sum_{l=1}^{(a-1) / 2} P_{2 l+1, n}(1) \zeta(2 l+1) .
$$

Une telle décomposition en $\zeta$ impairs suggère d'utiliser non plus un simple critère d'irrationalité comme le lemme 1 mais un critère d'indépendance linéaire, par exemple celui de Nesterenko [Ne2] :

Lemme 2. Soit $N$ réels $\theta_{1}, \theta_{2}, \ldots, \theta_{N}$. Supposons qu'il existe $N$ suites d'entiers $\left(p_{l, n}\right)_{n \geq 0}$ tels que :

i) $\left|\sum_{l=1}^{N} p_{l, n} \theta_{l}\right|=\alpha^{n+o(n)}$ avec $\alpha>0$;

ii) $\forall l=1, \ldots, N,\left|p_{l, n}\right| \leq \beta^{n+o(n)}$ avec $\beta>1$. 
Alors, dans ces conditions,

$$
\operatorname{dim}_{\mathbb{Q}}\left(\mathbb{Q} \theta_{1}+\mathbb{Q} \theta_{2}+\cdots+\mathbb{Q} \theta_{N}\right) \geq 1-\frac{\log (\alpha)}{\log (\beta)} .
$$

Un dénominateur commun aux $P_{2 l+1, n}(1)$ est $d_{n}^{a}$. Pour estimer $S_{n, a, r}(1)$, on remarque par exemple que

$$
\begin{aligned}
& S_{n, a, r}(z)=z^{-r n-1} n !^{a-2 r} \frac{\Gamma(r n+1)^{a+1} \Gamma((2 r+1) n+2)}{\Gamma((r+1) n+2)^{a+1}} \\
& \times{ }_{a+2} F_{a+1}\left(\begin{array}{c}
r n+1, \ldots, r n+1,(2 r+1) n+2 \\
(r+1) n+2, \ldots,(r+1) n+2
\end{array} \mid z^{-1}\right)
\end{aligned}
$$

est une fonction hypergéométrique "well-poised" ayant la réprésentation intégrale eulérienne (en $z=1$ )

(2) $S_{n, a, r}(1)=$

$$
\frac{((2 r+1) n+1) !}{n !^{2 r+1}} \int_{[0,1]^{a+1}}\left(\frac{\prod_{l=1}^{a+1} x_{l}^{r}\left(1-x_{l}\right)}{\left(1-x_{1} \cdots x_{a+1}\right)^{2 r+1}}\right)^{n} \cdot \frac{\mathrm{d} x_{1} \cdots \mathrm{d} x_{a+1}}{\left(1-x_{1} \cdots x_{a+1}\right)^{2}} .
$$

On en déduit en particulier l'existence et la non-nullité de la limite suivante, ainsi qu'une majoration simple

$$
\lim _{n \rightarrow+\infty}\left|S_{n, a, r}(1)\right|^{1 / n} \leq \frac{2^{r+1}}{r^{a-2 r}}
$$

La détermination explicite des coefficients de $P_{2 l+1, n}(1)$ permet leur estimation

$$
\limsup _{n \rightarrow+\infty}\left|P_{2 l+1, n}(1)\right|^{1 / n} \leq(2 r+1)^{2 r+1} 2^{a-2 r} .
$$

L'application du lemme 2 à la combinaison linéaire $d_{n}^{a} S_{n, a, r}(1)$ en les $\zeta$ impairs fournit une minoration explicite en fonction de $a$ impair et de $r$ de la dimension $\delta(a)$ du $\mathbb{Q}$ - espace vectoriel engendré par $1, \zeta(3), \zeta(5), \ldots, \zeta(a)$. Le choix $a=169$ et $r=10$, ainsi que le théorème d'Apéry, montre dans un premier temps le

Théorème 3. Il existe un entier $j$ impair, $5 \leq j \leq 169$ tel que $1, \zeta(3)$ et $\zeta(j)$ sont linéairement indépendants sur $\mathbb{Q}$.

Le choix $r=\left[a / \log ^{2}(a)\right]$ permet en outre d'obtenir la minoration asymptotique suivante ${ }^{5}$.

Théorème 4. Pour tout $\varepsilon>0$, il existe un entier $A(\varepsilon)$ tel que pour tout entier impair $a \geq A(\varepsilon)$, on $a$

$$
\delta(a) \geq \frac{1-\varepsilon}{1+\log (2)} \log (a) .
$$

${ }^{5}$ Si on remplace $(1-\varepsilon) /(1+\log (2))$ par $1 / 3$, on peut supposer $a \geq 3$. 
Corollaire 1. Une infinité des nombres $\zeta(2 n+1)$ sont irrationnels.

\section{Une approche vers l'irrationalité de $\zeta(5)$}

Suivant une démarche "d'extraction de facteurs communs" développée par Chudnovski [Ch], Rukhadze [Ru], Hata [Ha] (entre autres), Zudilin [Zu1] a raffiné le théorème 3 en remplaçant 169 par 145 . En se contentant de chercher l'irrationalité d'un tel $\zeta(j)$ directement, l'auteur [Ri2] a démontré le

Théorème 5. Au moins un des neuf nombres

$$
\zeta(5), \zeta(7), \zeta(9), \zeta(11), \zeta(13), \zeta(15), \zeta(17), \zeta(19), \zeta(21)
$$

est irrationnel.

Zudilin [Zu2] est parvenu à améliorer de manière spectaculaire ce résultat au moyen de la méthode arithmétique évoquée ci-dessus.

Théorème 6. Au moins un des quatre nombres

$$
\zeta(5), \zeta(7), \zeta(9), \zeta(11)
$$

est irrationnel.

Nous esquissons seulement la preuve du théorème 5 , celle du théorème 6 étant très technique. Considérons la série

$$
S_{n, a}(z):=n !^{a-6} \sum_{k=1}^{+\infty} \frac{1}{2} \frac{\mathrm{d}^{2}}{\mathrm{~d} t^{2}}\left((2 t+n) \frac{(t-n)_{n}^{3}(t+n+1)_{n}^{3}}{(t)_{n+1}^{a}}\right)_{\mid t=k} z^{-k}
$$

en remarquant que la dérivation induit un décalage dans la décomposition en polylogarithmes (comparer $U_{n}(z)$ et $V_{n}(z)$ au paragraphe 4). On a donc

$$
S_{n, a}(z)=P_{0, n}(z)+\sum_{l=1}^{a} P_{l, n}(z) \operatorname{Li}_{l+2}(1 / z)
$$

avec $d_{n}^{a+2} P_{l, n}(z) \in \mathbb{Z}[z]$. De nouveau, en supposant $a$ pair, pour tout $n$,

$$
S_{n, a}(1)=P_{0, n}(1)+\sum_{l=2}^{a / 2} P_{2 l-1, n}(1) \zeta(2 l+1),
$$

qui est une combinaison linéaire dont non seulement les $\zeta$ pairs ont disparu mais aussi $\zeta(3)$. En utilisant une généralisation immédiate du Lemme 1, on voit qu'il suffit de montrer que $S_{n, 20}(1) \neq 0\left({ }^{6}\right)$ et que $d_{n}^{22} S_{n, 20}(1)$ tend vers 0 . On notera qu'il n'y a pas lieu ici d'évaluer les coefficients des combinaisons linéaires : cela n'est utile que pour l'indépendance linéaire.

\footnotetext{
${ }^{6} \mathrm{cf}$. note (2).
} 
Comme pour $V_{n}(1)$, en l'absence d'intégrale de Beukers, une intégrale de Barnes fait l'affaire. En effet en posant

$$
\begin{aligned}
& J_{n}(i \pi):=\frac{(-1)^{n} n^{2}}{2 i \pi} n !^{14} \\
& \quad \times \int_{c+i \infty}^{c-i \infty}(2 z+1) \frac{\Gamma(n z)^{23} \Gamma(n-n z+1)^{3} \Gamma(n z+2 n+1)^{3}}{\Gamma(n z+n+1)^{23}} e^{i n \pi z} \mathrm{~d} z,
\end{aligned}
$$

on a $S_{n, 20}(1)=\operatorname{Re}\left(J_{n}(i \pi)\right)$ avec $0<c<1$. La formule de Stirling implique que

$$
J_{n}(i \pi)=i(-1)^{n+1}(2 \pi)^{9} n^{-8} \int_{L} g(z) e^{n w(z)} \mathrm{d} z \cdot\left(1+\mathrm{O}\left(n^{-1}\right)\right)
$$

avec

$$
g(z)=(2 z+1) \frac{\sqrt{1-z}^{3} \sqrt{z+2}^{3}}{\sqrt{z}^{23} \sqrt{z+1}^{23}}
$$

et

$$
\begin{aligned}
w(z)=23 z \log (z)-23(z+1) \log (z+1) \\
\quad+3(1-z) \log (1-z)+3(z+2) \log (z+2)+i \pi z .
\end{aligned}
$$

La méthode du col s'applique et après quelques calculs un peu laborieux, on arrive à la conclusion que

$$
\limsup _{n \rightarrow+\infty}\left|d_{n}^{22} S_{n, 20}(1)\right|^{1 / n} \approx e^{-0,02},
$$

ce qui prouve le théorème 5 .

\section{Une histoire hypergéométrique}

Revenons maintenant au paragraphe 4 et à la très surprenante valeur de la limite de $B_{n}(1)^{1 / n}$, qui coïncide avec celle de $\left|V_{n}(1)\right|^{1 / n}$. Cette cö̈ncidence est d'autant plus troublante que les quelques premières valeurs de $\alpha_{n}(1)$ sont entières et surtout égales aux nombres d'Apéry $A_{n}(1)$ introduits au paragraphe 4 , et de même semble-t-il $2 \delta_{n}(1)=D_{n}(1)$.

Puisque $\zeta(3)$ est irrationnel et que les suites $B_{n}(1)$ et $V_{n}(1)$ vérifient la même relation de récurrence à coefficients rationnels $\left({ }^{7}\right)$, on a bien $A_{n}(1)=$ $\alpha_{n}(1)$ pour tout entier $n$, c'est-à-dire la très improbable identité

$$
\sum_{k=0}^{n}\left(\begin{array}{l}
n \\
k
\end{array}\right)^{2}\left(\begin{array}{c}
n+k \\
n
\end{array}\right)^{2}=(-1)^{n} \sum_{j=0}^{n}\left(\begin{array}{c}
n \\
j
\end{array}\right)^{4}\left(\begin{array}{c}
n+j \\
n
\end{array}\right)\left(\begin{array}{c}
2 n-j \\
n
\end{array}\right)\left(\frac{n}{2}-j\right) H_{n, j}
$$

\footnotetext{
${ }^{7}$ Pour le voir, il suffit d'utiliser le programme Maple EKHAD de Zeilberger.
} 
où

$$
H_{n, j}=\sum_{k=1}^{n} \frac{1}{n+k-j}-\sum_{k=1}^{n} \frac{1}{k+j}-\sum_{\substack{k=0 \\ k \neq j}}^{n} \frac{4}{k-j}+\frac{1}{n / 2-j} .
$$

Ce raisonnement "circulaire" ne résout évidemment pas le problème de Nesterenko. Cependant, l'histoire ne s'arrête pas là. En effet, en travaillant sur la mesure d'irrationalité de $\zeta(3)$, Zudilin [Zu2] a rencontré la formule abracadabrantesque suivante, due à Bailey [Sl, p.142, 4.7.1.3]

$$
\begin{aligned}
& { }_{7} F_{6}\left(\begin{array}{ccc}
a, & 1+a / 2, \quad b, \quad c, \quad d, \quad e, \quad f \\
a / 2,1+a-b, 1+a-c, 1+a-d, 1+a-e, 1+a-f
\end{array} \mid 1\right) \\
& =\Gamma\left(\begin{array}{c}
1+a-b, \quad 1+a-c, \quad 1+a-d, 1+a-e, 1+a-f \\
a+1, b, c, d, 1+a-c-d, 1+a-b-d, 1+a-b-c, 1+a-e-f
\end{array}\right) \\
& \cdot \frac{1}{2 i \pi} \int_{L}^{\Gamma}\left(\begin{array}{c}
b+s, c+s, d+s, 1+a-e-f+s, 1+a-b-c-d-s,-s \\
1+a-e+s, \\
1+a-f+s
\end{array}\right) \mathrm{d} s
\end{aligned}
$$

avec la notation

$$
\Gamma\left(\begin{array}{l}
a_{1}, \ldots, a_{p} \\
b_{1}, \ldots, b_{q}
\end{array}\right)=\frac{\prod_{i=1}^{p} \Gamma\left(a_{i}\right)}{\prod_{j=1}^{q} \Gamma\left(b_{j}\right)}
$$

et où $L$ est un contour qui laisse certains pôles de l'intégrande à droite et d'autres à gauche, les paramètres étant choisis de sorte que les membres de gauche et de droite aient un sens simultanément.

Zudilin choisit $a=3 n+2$ et $b=c=d=e=f=n+1$ : dans ce cas, $L$ est une droite verticale orientée de $-i \infty$ à $+i \infty$ et comprise dans la bande $-n-1<\operatorname{Re}(s)<0$. Alors

$$
\begin{aligned}
& 2 B_{n}(1) \\
& =\frac{\Gamma(n+1)^{7} \Gamma(3 n+3)}{\Gamma(2 n+2)^{5}}{ }_{7} F_{6}\left(\begin{array}{r}
3 n+2,3 n / 2+2, n+1, \ldots, n+1 \\
3 n / 2+1,2 n+2, \ldots, 2 n+2
\end{array} \mid 1\right) \\
& =\frac{1}{2 i \pi} \int_{L} \frac{\Gamma(n+1+s)^{4} \Gamma(-s)^{2}}{\Gamma(2 n+2+s)^{2}} \mathrm{~d} s=\frac{1}{2 i \pi} \int_{L^{\prime}}\left(\frac{\Gamma(t)^{2} \Gamma(n+1-t)}{\Gamma(t+n+1)}\right)^{2} \mathrm{~d} t
\end{aligned}
$$

où, après le changement de variable $s=t-n-1, L^{\prime}$ est une droite comprise dans la bande $0<\operatorname{Re}(s)<n+1$. Or la dernière intégrale ci-dessus vaut exactement $V_{n}(1)$ et on démontre que $2 B_{n}(1)=V_{n}(1)$ sans utiliser cette fois de récurrence.

Néanmoins, si on sait donc démontrer de deux façons différentes que $2 A_{n}(1) \zeta(3)+D_{n}(1)=2 \alpha_{n}(1) \zeta(3)+2 \delta_{n}(1)$, on ne peut en déduire $\alpha_{n}(1)=$ $A_{n}(1) \in \mathbb{Z}$ et $2 d_{n}^{3} \delta_{n}(1)=d_{n}^{3} D_{n}(1) \in \mathbb{Z}$ qu'en utilisant l'irrationalité de $\zeta(3) \ldots$ Le problème de Nesterenko est donc toujours ouvert. 


\section{Des dénominateurs plus petits que prévu ?}

Le dénominateur de la série $B_{n}(1)$ n'est donc pas $d_{n}^{4}$ mais $d_{n}^{3}$ : ce phénomène n'est pas isolé puisque par exemple le dénominateur de la série $S_{n, 20}(1)$ au paragraphe 7 semble être expérimentalement $d_{n}^{21}$ et non $d_{n}^{22}$. Plus généralement, considérons la fraction rationnelle

$$
R_{n, a, b, c, d, r}(t)=n !^{a-2 b r}(2 t+n)^{c} \frac{(t-r n)_{r n}^{b}(t+n+1)_{r n}^{b}}{(t)_{n+1}^{a}}
$$

et la série

$$
S_{n, a, b, c, d, r}(z)=\sum_{k=1}^{+\infty} \frac{1}{d !} R_{n, a, b, c, d, r}^{(d)}(k) z^{-k}
$$

où $a, b, c, d, r$ sont des entiers tels que $r \geq 1, a>2 b r$ et $d \in\{0, \ldots, b-1\}$, ce qui assure la convergence de la série $S_{n, a, b, c, d, r}(z)$ pour tout $z \in \mathbb{C},|z|>1$ et sur $|z|=1$ si $n$ est assez grand. Pour simplifier les notations, la dépendance en les entiers $a, b, c, d$ et $r$ est maintenant omise. Il existe des polynômes $P_{l, n}(z)$ tels que

$$
S_{n}(z)=P_{0, n}(z)+\sum_{l=1}^{a} P_{l, n}(z) \mathrm{Li}_{l+d}(1 / z)
$$

et tels que pour tout $d \in\{0, \ldots, b-1\}$ et tout $l \in\{1, \ldots, a\}$,

$$
d_{n}^{a+d} P_{0, n}(z) \in \mathbb{Z}[z] \quad \text { et } \quad d_{n}^{a-l} P_{l, n}(z) \in \mathbb{Z}[z] .
$$

De plus, $P_{1, n}(1)=0$ et en supposant $a$ pair et $c$ impair, pour tout $l \geq 2$ pair, $P_{l, n}(1)=0$. Des calculs numériques suggèrent la conjecture suivante ${ }^{8}$.

Conjecture Dans les conditions de la définition de $S_{n}(z)$, si a est pair et $c$ impair, alors

$$
d_{n}^{a+d-1} P_{0, n}(1) \in \mathbb{Z}
$$

et pour tout $l \in\{3, \ldots, a-1\}$ impair,

$$
d_{n}^{a-l-1} P_{l, n}(1) \in \mathbb{Z} .
$$

Zudilin [Zu2] énonce une conjecture encore plus générale dont la démonstration est semble-t-il un passage obligé pour espérer améliorer le théorème 6 avec les techniques de [Zu1,2].

\footnotetext{
${ }^{8} \mathrm{Si} a$ est impair, on a une conjecture similaire pour $P_{l, n}(-1), l \geq 0$ pair.
} 


\section{En conclusion}

Un certain nombre de questions se posent naturellement. En voici quelques-unes et les réponses que l'on peut éventuellement faire.

Est-il possible d'établir par ces méthodes une sorte de théorème de Dirichlet "Une infinité de valeurs irrationnelles parmi les $\zeta(a n+b)$ " ? Bien qu'il s'agisse d'un théorème hautement plausible, et en dehors de quelques cas triviaux et du corollaire 1 , c'est un problème ouvert.

Étant donné un entier impair $a \geq 3$, est-il possible de déterminer un entier $\alpha$ tel que l'ensemble $\{a, \ldots, a+\alpha\}$ contienne un entier $j$ impair tel que $\zeta(j)$ soit irrationnel ? Oui. Zudilin [Zu1] montre que l'on peut choisir $\alpha=7 a-17$.

Est-il maintenant possible de montrer l'irrationalité de la constante de Catalan $G=\sum_{n \geq 0}(-1)^{n} /(2 n+1)^{2}$ ? Non, c'est toujours un problème ouvert, en particulier parce que les dénominateurs des approximations sont "trop gros". Néanmoins on se réferera à [RZ] pour les analogues des théorèmes 4 et 6 pour la fonction $\beta(s)=\sum_{n>0}(-1)^{n} /(2 n+1)^{s}$, dont ce sont les valeurs paires qui posent problème.

Quid des multizêtas (MZV) ? C'est en effet une extension naturelle. Cependant, s'il est facile d'écrire des séries multiples dont on sait qu'elles ne peuvent pas être décomposées en autre chose que des $\mathrm{MZV}$, décrire cette décomposition ne semble pas si évident. Par ailleurs, les combinaisons feront naturellement apparaître des MZV s'exprimant linéairement à l'aide des $\zeta(s)$ : il semble donc important d'éliminer a priori ces valeurs pour que la minoration de la dimension de certains espaces vectoriels ne soit pas triviale.

Les séries $B_{n}(z), S_{n, a, r}(z), S_{n, 2}(z)$ (et toutes celles que l'on peut former sur ce modèle) sont-elles solutions uniques de problèmes d'approximation de type Padé comme les séries $S_{n}(z), U_{n}(z)$ et $V_{n}(z)$ ? Un cas simple est abordé dans [Ri3, p. 53] mais une théorie générale reste à construire.

Existe-t-il une identité de Bailey généralisée qui permettrait de démontrer les conjectures du paragraphe 9 et de [Zu2] ? Dans ce cas, on pourrait abor$\operatorname{der} \zeta(5)$, via la méthode de Rhin et Viola [RV1,2], en faisant agir un groupe sur des intégrales d'Euler ou de Barnes.

Note ajoutée sur les épreuves. Contrairement à l'affirmation p. 355, il existe une démonstration simple du passage direct de la série $V_{n}(1)$ à l'intégrale triple de Beukers : elle est esquissée dans un article en préparation de l'auteur, Valeurs de la fonction zêta de Riemann aux entiers. Dans [Zu3], Zudilin montre directement à l'aide de l'algorithme de Zeilberger que les nombres $\alpha_{n}(1)$ et $A_{n}(1)$ considérés au paragraphe 8 sont égaux pour tout entier $n \geq 0$, sans supposer l'irrationalité de $\zeta(3)$ : ceci répond en un certain sens au problème de Nesterenko. Enfin, la question posée 
ci-dessus sur l'existence d'un lien entre nos séries hypergéométriques et l'approximation de Padé a été résolue par l'affirmative dans [FR].

\section{Bibliographie}

[Apl] R. APÉRY, Irrationalité de $\zeta(2)$ et $\zeta(3)$. Astérisque 61 (1979), 11-13.

[Ap2] R. APÉRY, Interpolation de fractions continues et irrationalité de certaines constantes. Bulletin de la section des sciences du C.T.H.S III (1981), 37-53.

[Ba] K. BALL, communications personnelles du 17/12/1999 et du 04/01/2000.

[BR] K. BALL, T. RIVOAL, Irrationalité d'une infinité de valeurs de la fonction zêta aux entiers impairs. Invent. Math. 146 (2001), 193-207.

[Bel] F. Beukers, $A$ note on the irrationality of $\zeta(2)$ and $\zeta(3)$. Bull. Lond. Math. Soc. 11 (1979), 268-272.

[Be2] F. BEUkers, The values of Polylogarithms. Topics in classical number theory, Vol. I, II (Budapest, 1981), 219-228, Colloq. Math. Soc. János Bolyai, 34, North-Holland, Amsterdam, 1984.

[Be3] F. Beukers, Padé approximations in Number Theory. Padé approximation and its applications, Amsterdam 1980 (Amsterdam, 1980), pp. 90-99, Lecture Notes in Math., 888, Springer, Berlin-New York, 1981.

[Ch] G. V. Chudnovski, On the method of Thue-Siegel. Ann. of Math. 117 (1983), 325-382.

[Coh] H. CoHEN, Démonstration de l'irrationalité de $\zeta(3)$ (d'après Apéry). Séminaire de Théorie des Nombres de Grenoble (1978), VI.1-VI.9.

[Cop] E. T. Copson, Asymptotic expansions. Cambridge University Press, 1967.

[Di] J. Dieudonné, Calcul infinitésimal. Collection "Méthodes", Hermann, 1980.

[Di] S. Fischler, T. Rivoal, Approximants de Padé et séries hypergéométriques équilibrées, à paraître à J. Math. Pures Appl. (2003).

[Gu] L. A. GUTNIK, The irrationality of certain quantities involving $\zeta(3)$. Russ. Math. Surv. 34 (1979), no. 3, 200. En russe dans Acta Arith. 42 (1983), 255-264.

[Ha] M. HATA, Legendre type polynomials and irrationality measures. J. Reine Angew. Math. 407 (1990), 99-125.

[Hu] M. HUTTNER, Équations différientielles fuchsiennes; Approximations du dilogarithme, de $\zeta(2)$ et $\zeta(3)$. Pub. IRMA. Lille, 1997.

[Li] F. LindemanN, Über die Zalh $\pi$, Math. Ann. 20 (1882), 213-225.

[Nel] YU. V. Nesterenko, On the linear independence of numbers. Mosc. Univ. Math. Bull.40 (1985), 69-74, traduction de Vest. Mosk. Univ. Ser. I (1985), 46-54.

[Ne2] Yu. V. Nesterenko, A few remarks on $\zeta(3)$, Math. Notes 59 (1996), no. 6, 625-636.

[Ni] E. M. Nikishin, On the irrationality of the values of the functions $F(x, s)$. Mat. Sbornik 37 (1979), no. 3, 381-388.

[Nø] N. E. NøRLUND, Hypergeometric functions. Acta Math. 94 (1955), 289-349.

[Pr] M. PrÉvost, A new proof of the irrationality of $\zeta(3)$ using Padé approximants. J. Comp. Appl. Math. 67 (1996), 219-235.

[RV1] G. RhiN, C. VIolA, On a permutation group related to $\zeta(2)$. Acta Arith. 77 (1996), 23-56.

[RV2] G. Rhin, C. Viola, The group structure for $\zeta(3)$. Acta Arith. 97 (2001), 269-293.

[Ri1] T. RIVOAL, La fonction zêta de Riemann prend une infinité de valeurs irrationnelles aux entiers impairs. C. R. Acad. Sci. Paris 331 (2000), 267-270.

[Ri2] T. Rivoal, Au moins un des neuf nombres $\zeta(5), \zeta(7), \ldots, \zeta(21)$ est irrationnel. Acta Arith. 103 (2002), 157-167.

[Ri3] T. Rivoal, Propriétés diophantiennes des valeurs de la fonction zêta aux entiers impairs. Thèse de doctorat, Université de Caen, 2001.

[RZ] T. RIVOAL, W. ZUDILIN, Diophantine properties of numbers related to Catalan's constant, à paraître à Math. Ann. (2003).

[Ru] E. A. RUKHADzE, A lower bound for the approximation of $\ln (2)$ by rationals numbers. Vestnik Moskov. Univ. Ser I Mat. Mekh 6 (1987), 25-29 (en russe).

[Sl] L. J. Slater, Generalized Hypergeometric Functions. Cambridge University Press, 1966. 
[So] V. N. Sorokin, Apéry's Theorem. Mosc. Univ. Math. Bull. 53 (1998), no. 3, 48-52.

[VdP] A. VAN DER POORTEN, A proof that Euler missed ... Apéry's proof of the irrationality of $\zeta(3)$. Math. Intellig. 1 (1979), 195-203.

[Wa] M. WALDSCHMIDT, Valeurs zêtas multiples. Une introduction. J. Théor. Nombres Bordeaux 12 (2000), 581-595.

[Zu1] W. Zudilin, Irrationality of values of zeta-function, à paraître à Izv. Ross. Akad. Nauk. Ser. Mat.

[Zu2] W. ZuDILIN, Arithmetic of linear forms involving odd zeta values, prépublication, Moscow Lomonosov State University, 2001.

[Zu3] W. Zudilin, An elementary proof of Apéry's theorem, (2002) ; http://front.math.ucdavis.edu/math.NT/0202159

Tanguy RIVOAL

Institut de Mathématiques de Jussieu CNRS UMR 7586

Théorie des nombres, case 247

175, rue du Chevaleret

75013 Paris

France

E-mail : rivoal@math.jussieu.fr et Laboratoire de Mathématiques Nicolas Oresme CNRS UMR 6139,

Université de Caen

BP 5186

14032 Caen cedex

France

E-mail : rivoal@math.unicaen.fr 